\title{
Um Estudo Empírico sobre os Benefícios da Procura e do Uso da Internet como Fonte de Informações
}

\author{
José Mauro da Costa Hernandez
}

\section{Resumo}

Este estudo replica estudos anteriores sobre os benefícios da procura de informações e introduz a Internet como fonte de informações para investigar se seus usuários obtiveram mais benefícios na compra de automóveis novos que os não usuários. Os resultados, além de terem permitido a identificação de amplo conjunto de variáveis que determinaram o tempo de procura, também levaram à conclusão que os compradores que utilizaram a Internet como fonte de informações investiram mais tempo na procura do que os compradores que não a utilizaram. Este resultado provavelmente é decorrente da diferença entre os perfis dos dois grupos (os usuários da Internet são mais jovens, possuem mais anos de educação formal e têm renda superior, características geralmente associadas a pessoas que investem mais tempo em procura). Quando os dois grupos foram analisados em conjunto, verificou-se que o tempo investido em procura não esteve associado positivamente com a obtenção de maiores descontos na compra de automóveis novos. No entanto, ao analisarse os dois grupos separadamente, constatou-se que o tempo de procura esteve positivamente associado com a obtenção de maiores descontos para o grupo de usuários da Internet, mas não para o grupo de não usuários. Discute-se os resultados obtidos e sugere-se algumas modificações no modelo teórico.

Palavras-chaves: procura de informações; automóveis; compra eletrônica; Internet; econometria.

\begin{abstract}
This study replicates previous studies focusing on consumer information search benefits and introduces the Internet as a source of information to investigate whether its users obtained more benefits in the purchase of new automobile than non-users. The results, besides allowing the identification of a large set of search determinants, also permitted to conclude that buyers that used the Internet as a source of information spent more time searching than buyers that did not use the Internet. This finding is likely to result from the different search profiles of both groups (Internet users are younger, more educated, and have higher income, characteristics usually associated with more search). When both groups were analyzed together, it was found that time spent on search was not positively associated with higher discounts in the purchase of the new automobile. However, when the two groups were separated and analyzed, it was found that time spent on search was positively associated with higher discounts for the group of Internet users but not for the group of non-users. Findings are discussed and modifications in the theoretical model are suggested.
\end{abstract}

Key words: search of information; cars; electronic shopping; Internet; econometry. 


\section{INTRODUÇÃO}

A fase de busca de informações do processo de decisão de compra do consumidor tem sido objeto de inúmeros estudos desde a publicação do artigo de George Stigler em 1961. Com o crescimento do volume de vendas ao consumidor realizadas por meio da Internet, especialmente nos Estados Unidos, a utilização da Internet como fonte de informações durante a fase de procura também passou a receber mais atenção (Klein e Ford, 2000; Ratchford, Talukdar e Lee, 2000).

Enquanto parece estar bem estabelecido na literatura que o maior investimento em procura está positivamente associado à obtenção de melhores preços, muito pouco se sabe sobre os benefícios da utilização da Internet como fonte de informações durante este processo.

Recentemente, alguns artigos têm apontado que o mercado eletrônico é mais eficiente que o mercado convencional (para uma revisão destes estudos, ver Smith, Bailey e Brynjolfsson [1999]) e uma das razões freqüentemente apontada para este resultado deriva da redução do custo da procura (por exemplo, em Bakos [1997]). Devido às suas características, a Internet permite aos seus usuários obter rápida e eficientemente grande quantidade de informações sobre as diferentes ofertas do mercado, facilitando a sua tarefa de encontrar os menores preços.

Portanto seria razoável supor que os compradores que utilizam a Internet durante o processo de procura tenham mais e melhores informações do que aqueles que não a utilizam, tornando-os mais aptos a conseguir melhores preços e investir menos tempo em procura.

Este estudo tem dois propósitos: o primeiro é replicar estudos anteriores sobre os benefícios da procura (por exemplo, o de Punj e Staelin [1983] e o de Ratchford e Srinivasan [1993]) e o segundo é verificar se consumidores que utilizam a Internet como fonte de informações são mais eficientes no processo de procura do que consumidores que não utilizam a Internet ${ }^{(1)}$.

Para cumprir os objetivos propostos, este estudo utiliza dados coletados em uma amostra de compradores norte-americanos de carros novos. Duas são as razões para a escolha de automóveis como objeto de estudo: a primeira razão é que já existe um grande número de artigos sobre procura que utilizou automóveis como foco de estudo, facilitando a comparação; a segunda razão é que, embora a Internet ainda não se tenha consolidado como canal de vendas de automóveis 
novos $^{(2)}$, em 2000 cerca de $41 \%$ dos compradores de automóveis novos nos Estados Unidos a utilizaram durante o processo de procura (Time, 2001).

Este estudo está estruturado em sete seções, assim distribuídas: a primeira seção contém uma breve revisão da literatura juntamente com as hipóteses que guiaram o estudo; a segunda seção descreve o modelo teórico utilizado para testar as hipóteses; a terceira seção discute as variáveis incluídas neste estudo e descreve as hipóteses sobre o efeito de cada uma destas variáveis no tempo de procura e nos descontos obtidos na compra de automóveis novos; a quarta seção descreve a metodologia de coleta dos dados; a quinta seção relata os resultados obtidos neste estudo; a sexta seção discute estes resultados e a última seção conclui o estudo, apontando as limitações e direções para novas pesquisas.

\section{REVISÃo dA LiteratuRA}

Este estudo se insere num amplo conjunto de literatura em marketing que tem investigado o processo de procura de informações, que corresponde ao estágio do processo de decisão de compra do consumidor, no qual ele busca ativamente coletar e integrar informações, tanto interna quanto externamente, antes de tomar uma decisão (Schmidt e Spreng, 1996).

O primeiro grupo de literatura relevante para este estudo refere-se aos benefícios da procura (Newman e Staelin, 1972; Carlson e Gieseke, 1983; Punj e Staelin, 1983; Beatty e Smith, 1987; Ratchford e Srinivasan, 1993; Urbany, Dickson e Kalapurakal, 1996). Estes estudos investigaram tanto os fatores que determinam o tempo investido em procura quanto os benefícios obtidos neste processo.

O paradigma dominante nesta literatura originou-se num artigo de Stigler (1961), segundo o qual os consumidores procuram por melhores alternativas, enquanto o custo marginal de uma unidade adicional de procura for inferior ao benefício marginal esperado com a obtenção de melhores alternativas.

Embora os determinantes da procura sejam muitos e variados (Beatty e Smith [1987], por exemplo, identificaram aproximadamente sessenta variáveis que haviam sido empiricamente estudadas como determinantes da procura), uma das principais conclusões deste grupo de estudos é que o maior tempo investido em procura está associado à obtenção de menores preços. A seguinte hipótese pode ser formulada com base em resultados de estudos anteriores:

H1: Tempo investido em procura está positivamente associado à obtenção de melhores preços. 
O segundo grupo de literatura relevante refere-se a um grupo emergente na literatura de marketing que tem analisado o comportamento dos consumidores em mercados eletrônicos (Bakos, 1997; Lee, 1998; Brynjolfsson e Smith, 2000; Degeratu, Rangaswamy e Wu, 2000; Lynch e Ariely, 2000). De forma geral, estes estudos concluíram que os mercados eletrônicos são mais eficientes do que os mercados convencionais e apontam a redução do custo da procura como um dos fatores responsáveis por esta maior eficiência.

De acordo com a teoria econômica, quanto maior a assimetria de informações entre compradores e vendedores, maior o custo de procura do comprador, implicando em preços superiores ao custo marginal na situação de equilíbrio (Varian, 1984). Devido às suas características, como interatividade, instantaneidade e alta capacidade de armazenamento de informações, a Internet proporciona aos seus usuários grande quantidade de informações a baixo custo, facilitando a sua tarefa de encontrar alternativas superiores àquelas já consideradas e reduzindo o risco associado à eliminação de alternativas inferiores.

Desta forma, mantidos os demais fatores constantes, seria esperado que os compradores que utilizam a Internet como fonte de informações sejam mais eficientes do que aqueles que não a utilizam.

Pode-se pensar eficiência em dois sentidos: tempo e dinheiro (no presente caso, menores preços). Se os usuários da Internet conseguem obter mais informações a menores custos, é de se supor que eles estejam mais aptos a conseguirem menores preços. Por exemplo, Morton, Zettelmeyer e Risso (2000) descobriram que os preços pagos por consumidores que obtiveram referências de concessionárias por meio do Website Autobytel.com eram aproximadamente 2\% inferiores ao preços pagos por consumidores que não utilizaram este serviço; os resultados deste estudo também indicaram que os consumidores que utilizaram este serviço de referência fizeram melhores compras do que pelo menos $61 \%$ dos consumidores que não utilizaram nenhum serviço de referência.

No entanto há que se considerar a habilidade do comprador na utilização da informação. É provável que pessoas com mais experiência ou maior conhecimento do mercado de automóveis tenham maior capacidade cognitiva no processamento de novas informações do que pessoas com menos experiência ou menor conhecimento (Brucks, 1985). A utilização da Internet, por si só, não pode garantir que a informação será utilizada eficientemente.

Mesmo considerando estas duas posições conflitantes, a seguinte hipótese é formulada:

H2: Consumidores que utilizam a Internet como fonte de informações conseguem melhores preços do que consumidores que não utilizam a Internet. 
Da mesma forma, se a Internet proporciona mais rapidez no acesso a mais e melhores informações, também seria razoável supor que os seus usuários tenham que investir menos tempo em procura. Por exemplo, ao invés de ir pessoalmente ou telefonar para diversas concessionárias, pedindo cotações, o usuário da Internet poderia conseguir todas as informações desejadas diretamente dos Websites dos fabricantes de automóveis ou de seus concessionários sem sair de casa.

Entretanto há que se fazerem duas considerações a este respeito. A primeira diz respeito à habilidade do comprador na utilização da Internet como fonte de informações. Usuários mais experientes têm maior facilidade em descartar informações irrelevantes e, desta forma, estariam mais aptos que usuários menos experientes para obter as vantagens que a Internet oferece (Ratchford, Talukdar e Lee, 2000).

A segunda refere-se às diferenças individuais de usuários e não usuários da Internet: usuários da Internet ainda são mais jovens, têm mais anos de educação formal e renda superior (U.S. Census Bureau, 200?). O perfil deste grupo de pessoas corresponde ao perfil das pessoas que procuram mais informações: os mais jovens, por terem menos experiência, dependem mais de fontes externas de informações do que fontes internas, ao contrário das pessoas mais velhas (Ratchford, 1982); as pessoas com mais anos de educação formal, por terem capacidade de utilizar a informação mais eficientemente (Ratchford, 1982); e, finalmente, as pessoas que têm renda superior por terem um conjunto de preferências maior e mais complexo (Ratchford, Talukdar e Lee, 2000).

Da mesma forma, embora sejam consideradas estas duas visões opostas, a seguinte hipótese é formulada:

H3: Consumidores que utilizam a Internet como fonte de informações investem menos tempo em procura do que consumidores que não utilizam a Internet.

\section{Modelo Teórico}

Objetivando testar as hipóteses formuladas na seção anterior e considerando a similaridade de objetivos, adotou-se como base o modelo teórico desenvolvido por Ratchford e Srinivasan (1993). Entretanto, devido à natureza das variáveis utilizadas naquele estudo, algumas alterações no modelo foram necessárias.

De acordo com a teoria econômica, o consumidor é visto como um ser racional que deve decidir como alocar seu tempo, dinheiro e esforço na produção de utilidade (Marmostein, Grewal e Fishe, 1992). Vista desta forma, a atividade de 
procura compete com outras atividades, como lazer e trabalho, pelo tempo limitado do consumidor.

No modelo tradicional, a utilidade obtida pelo consumidor com a procura é geralmente mensurada em termos econômicos. A literatura sugere, entretanto, que o consumidor também obtém retornos não econômicos, como satisfação com a atividade de compra (Marmostein, Grewal e Fishe, 1992), com o fato de se fazer uma boa compra (Ratchford e Srinivsan, 1993) ou ainda de estar bem informado e poder ajudar os amigos (Feick e Price, 1987). No entanto medidas econômicas têm sido mais utilizadas do que medidas de satisfação, uma vez que o preço pago (ou medidas correlatas) pela mercadoria é mais facilmente observável do que a satisfação obtida com a compra.

Assim, o processo de procura pode ser visto como função do investimento de tempo e, eventualmente, outros recursos, na produção de menores preços, sugerindo a seguinte relação:

$\mathrm{P}=\mathrm{P}(t, \mathrm{R}, \mathrm{C})$

na qual $\mathrm{P}$ = preço pago pelo produto; $t=$ tempo investido na procura por menores preços; $\mathrm{R}$ = características do veículo e da concessionária; e C = características do comprador. A partir desta relação, Ratchford e Srinivasan (1993) derivaram uma equação na qual a margem bruta obtida pela concessionária varia inversamente com a eficiência no processo de procura do consumidor e com o tempo investido neste processo, relação esta que é moderada pela concentração da oferta de preços. A margem bruta obtida pela concessionária é uma medida do retorno econômico do comprador uma vez que, quanto menor esta margem, menor o preço pago pelo veículo.

Neste estudo, ao contrário do estudo de Ratchford e Srinivasan (1993), tanto os preços efetivamente pagos pelos veículos quanto o montante de descontos obtidos pelos compradores estavam disponíveis; por outro lado, não estavam disponíveis informações relativas às concessionárias, como volume de estoques e custos operacionais. Portanto, considerando as informações disponíveis e os objetivos deste estudo, sugere-se a seguinte relação:

$\mathrm{D}=D(t, \mathrm{I}, \mathrm{V}, \mathrm{C})$

na qual $D=$ desconto obtido na compra; $t=$ tempo investido na procura; $\mathrm{I}=$ variável que indica se o comprador consultou a Internet ou não; $\mathrm{V}=$ características do veículo; e C = características do comprador.

Seguindo o modelo proposto por Ratchford e Srinivasan (1993), o seguinte sistema de equações pode ser derivado: 
(1) $\log D^{*} \quad=\quad \alpha_{0}+\alpha_{1} \log t+\alpha_{2} I+V \Gamma_{1}+C \Pi_{1}+\varepsilon_{1}$

(1a) $\log D \quad=\quad \log D^{*} \quad$ se $\log D^{*}>0$

$$
=0 \quad \text { caso contrário }
$$

(2) $\log t \quad=\quad \beta_{0}+\beta_{1} \log D+\beta_{2} I+V \Gamma_{2}+C \Pi_{2}+\varepsilon_{2}$

no qual $D, t, I, V$, e $C$ correspondem às variáveis definidas acima e $\Gamma 1, \Pi 1, \Gamma 2 \mathrm{e}$ $\Pi 2$ são os vetores de coeficientes estimados.

Duas observações importantes devem ser feitas sobre o sistema de equações acima. Em primeiro lugar, observa-se que $D$ e $t$ são interdependentes, o que pode ser explicado pela distribuição da oferta de preços que o consumidor encontra durante o processo de procura: ao perceber que há grande variação nos preços, o consumidor passa a acreditar que vale a pena continuar procurando por menores preços (maiores descontos); quando a distribuição de preços é homogênea, entretanto, o consumidor percebe que não será fácil conseguir menores preços (maiores descontos), o que o desestimula a continuar procurando. Uma das principais implicações da interdependência entre $D$ e $t$ é que as equações (1) e (2) não podem ser estimadas isoladamente.

A segunda observação refere-se à natureza da distribuição dos descontos; uma vez que nem todos os compradores conseguem descontos $(D=0), D$ é uma variável dependente limitada, como mostra a formulação da equação (1).

Para resolver o sistema de equações simultâneas acima, adotou-se o procedimento sugerido por Nelson e Olson (1978). Estes autores sugerem um procedimento análogo àquele utilizado em equações simultâneas sem variáveis dependentes limitadas por meio do Método de Mínimos Quadrados em Dois Estágios. Aplicar este procedimento no caso acima consiste em utilizar o conjunto de variáveis exógenas $(V$ e $C$ ) na criação de variáveis instrumentais $(\hat{D}$ e $\hat{t})$ e depois substituir estas variáveis pelos seus pares no lado direito das equações. Especificamente, os coeficientes das equações (1) foram estimados, utilizando-se Análise Tobit (Tobin, 1958) para variáveis censuradas por meio do Método de Máxima Probabilidade, enquanto os coeficientes da equação (2) foram estimados normalmente pelo Método de Mínimos Quadrados. Nelson e Olson (1978) sugerem que os coeficientes obtidos desta forma são consistentes e assimptóticos. 


\section{VARIÁveis IncLuídas no Estudo}

\section{Variáveis Independentes}

A literatura sobre procura é a melhor fonte de informações sobre os fatores que determinam o tempo investido em procura (Beatty e Smith, 1987; Srinivasan e Ratchford, 1991; Ratchford e Srinivasan, 1993). Ao se incluir o maior número possível de variáveis que possam determinar o montante de tempo investido em procura e na obtenção de melhores preços, consegue-se controlar efeitos espúrios, além de se compreender melhor este complexo fenômeno.

Com base em resultados de estudos anteriores e no conhecimento do mercado de automóveis novos, são descritas a seguir as variáveis incluídas neste estudo, o sinal esperado do efeito de cada variável sobre as variáveis dependentes $D$ e $t$, a justificativa para a inclusão de cada variável no modelo e a forma de mensuração de cada uma no estudo.

\section{Características do Consumidor}

Em princípio, qualquer característica do consumidor que por hipótese o torne mais eficiente na procura deveria ser incluída nas equações (1) e (2), conforme explicitado em seguida.

- Grau de escolaridade $(+,+)^{(3)}$ : maior grau de escolaridade pode implicar maior capacidade de coletar e analisar informações, o que conduziria a menos tempo de procura; por outro lado, por serem mais eficientes na obtenção e análise de informações, pessoas com maior grau de escolaridade têm um custo marginal de procura inferior, o que poderia levá-las a investir mais tempo em procura (Stigler, 1961; Ratchford, 1982). Assim, a hipótese deste estudo é que o maior grau de escolaridade conduzirá a mais procura e, conseqüentemente, a maiores descontos. Esta variável foi mensurada em número de anos de estudo formal e posteriormente transformada em duas variáveis dummy: POSGRAD indica indivíduos com mais de 17 anos de estudo formal (corresponderia no Brasil a estudos de pós-graduação) e GRADUADO indica indivíduos com mais de 13 e menos de 17 anos de estudo formal (corresponderia no Brasil a estudos de curso superior).

- Conjuntos evocados de modelos de automóveis e concessionárias (+,?): quanto maiores os conjuntos evocados de modelos de automóveis e de concessionárias, mais informações o consumidor terá que reunir para julgar as dife- 
rentes possibilidades e mais tempo terá que investir na procura. É impossível prever, por outro lado, o efeito desta variável sobre os descontos obtidos, já que mais tempo de procura, diluído entre vários modelos/concessionárias, pode resultar em menos procura por modelo/concessionária. Estes dados foram obtidos solicitando aos respondentes que informassem o número de modelos de veículos (MODVEIC) e concessionárias (CONCESS) considerados antes da compra.

. Número de carros adquiridos nos últimos dez anos (-,+): quanto maior a experiência do consumidor na aquisição de veículos novos ou usados, maior o seu conhecimento do mercado de automóveis e, supostamente, maior o seu estoque de informações. Desta forma, ele precisaria investir menos tempo em procura e poderia usar a sua experiência para obter maiores descontos. Para se obter esta variável, foi solicitado aos respondentes informarem quantos veículos novos e usados eles haviam adquirido nos últimos 10 anos (NCARDEZ).

. Número de concessionárias nos últimos dez anos (-,+): da mesma forma que o item anterior, quanto maior o número de diferentes concessionárias das quais o comprador adquiriu automóveis nos últimos 10 anos, maior a sua experiência na aquisição de veículos e, portanto, maior a sua eficiência no processo de procura e na obtenção de descontos (NCONCDEZ).

- Tempo decorrido entre o início do processo de compra e a compra efetiva $(+,+)$ : quanto mais tempo o consumidor leva entre o momento em que ele se decide pela compra e o momento em que ele efetivamente compra o veículo, mais tempo ele deverá investir em procura e, supostamente, maior a probabilidade de obter maiores descontos. Esta variável é a resposta à pergunta: “Quando você pensou pela primeira vez em adquirir um novo carro?”. Foi mensurada numa escala de 5 itens, sendo então recodificada para compor quatro variáveis dummy: QUANDO1 (menos de 1 mês antes da compra), QUANDO2 (entre 1 e 3 meses antes da compra), QUANDO3 (entre 3 e 6 meses antes da compra) e QUANDO4 (entre 6 e 12 meses antes da compra).

- Certeza em relação à marca do veículo (-,-): o maior grau de certeza em relação à marca desejada implica menor tempo de procura e, devido à lealdade, menor sensibilidade ao preço do veículo a ser adquirido. Esta variável é a resposta à pergunta: “A partir do momento em que você considerou seriamente a aquisição de novo veículo, você já sabia de que fabricante iria adquirir o veículo?”. Ela foi mensurada numa escala de 3 itens, transformada posteriormente em duas variáveis dummy: CERTOMSIM (definitivamente sim) e CERTOMNAO (definitivamente não). 
. Certeza em relação à concessionária $(-,+)$ : maior certeza em relação à concessionária pode implicar menos tempo de procura. Por outro lado, é provável que a certeza em relação à concessionária resulte de lealdade decorrente da certeza de conseguir maiores descontos. Esta variável é a resposta à pergunta: "A partir do momento em que você considerou seriamente a aquisição de novo veículo, você já sabia de que concessionária iria adquirir o veículo?”. Esta foi mensurada numa escala de 3 itens, transformada posteriormente em duas variáveis dummy: CERTOFSIM (definitivamente sim) e CERTOFNAO (definivamente não).

A rigor, não existiriam razões para se conjecturar que variáveis demográficas influam significativamente sobre o tempo investido em procura ou sobre os descontos obtidos numa compra. Variáveis como idade, educação e sexo já foram utilizadas como medidas de eficiência de procura (por exemplo, por Blaylock e Smallwood [1987]), embora, com exceção da variável educação, os resultados de estudos anteriores não permitam nenhuma conclusão definitiva.

Em estudos econométricos, Ayres (1991) e Ayres e Siegelman (1995) demonstraram que homens brancos conseguiram ofertas de preços melhores por automóveis novos do que mulheres e homens negros; Goldberg (1996), entretanto, não encontrou nenhuma relação entre características demográficas e preços na compra de carros novos.

Tendo em vista os resultados controversos dos estudos mencionados acima, decidiu-se pela inclusão de algumas variáveis demográficas neste estudo, discriminadas a seguir.

. Idade (-,+): assumiu-se que mais idade se reflete em maior experiência e, portanto, menor necessidade de se investir em procura e maior probabilidade de conseguir maiores descontos. Esta variável foi mensurada numa escala de 5 pontos e as respostas foram transformadas em quatro variáveis dummy: IDADE30, IDADE3140, IDADE4150, IDADE5160.

- Sexo (?,?): não é possível prever o efeito do sexo do comprador sobre o tempo de procura ou sobre os descontos obtidos na compra de veículos novos. Foi criada uma variável (HOMEM) para indicar pessoas do sexo masculino.

- Estado civil (?,?): também não é possível prever o efeito do estado civil sobre o tempo de procura ou sobre os descontos obtidos na compra de veículos novos. Foi criada uma variável para distinguir os homens casados (CASADO) dos homens que não estavam casados no momento da pesquisa (solteiros, divorciados e viúvos). 


\section{Características do Veículo}

. Número de opcionais (?,-): supostamente, quanto maior o número de opcionais desejados pelo consumidor, mais tempo ele teria que investir para chegar a um bom negócio, uma vez que os opcionais são utilizados pela indústria para valorizar os veículos e diminuir os descontos; por outro lado, como o número de opcionais é em grande parte decidido pelos fabricantes e os carros norte-americanos normalmente já vêm equipados com grande número de opcionais, encontrar veículos com menos opcionais pode ser tarefa extremamente difícil. Por sua vez, mais opcionais pode implicar menores descontos. Foi solicitado aos respondentes informarem quais, em uma lista de 12 exemplos de opcionais, o carro adquirido possuía. As doze variáveis dummy obtidas foram consolidadas em uma única variável (OPTOTAL).

- Modelo do veículo (?,?): é possível que seja mais fácil encontrar informações para alguns modelos e mais difícil para outros. Por exemplo, para modelos de marcas com maior número de concessionárias, o tempo de procura pode ser menor do que para modelos de marcas com menor número de concessionárias, devido ao acesso mais fácil aos showrooms. Da mesma forma, é possível que seja mais fácil barganhar com as concessionárias de alguns modelos. Por exemplo, as concessionárias da marca Saturn reconhecidamente não oferecem descontos, seguindo a política do fabricante. Desta forma, optou-se por utilizar o modelo do automóvel como controle das diferenças de preços entre os veículos. Foi solicitado ao respondente informar o modelo e o fabricante do veículo adquirido e para cada modelo foi criada uma variável dummy.

\section{- Variável que indica se o veículo usado foi utilizado na transação ou não} (?,-): os resultados de Goldberg (1996) são consistentes com a hipótese de que as concessionárias dão menos descontos quando o carro usado é envolvido na transação. Provavelmente, as concessionárias supervalorizam o carro usado e, em decorrência, dão descontos menores na compra do veículo novo. É difícil prever, entretanto, a influência que esta variável poderia ter sobre o tempo de procura. Para se obter esta variável, foi solicitado aos respondentes informarem se eles haviam dado um carro usado como parte do pagamento (USADO).

\section{Variáveis Dependentes}

. Descontos obtidos na compra do veículo novo: foi solicitado aos respondentes informarem os descontos obtidos tanto do fabricante ${ }^{(4)}$ quanto da concessionária $(D)$.

. Tempo investido na procura: foi solicitado aos respondentes informarem 
quanto tempo (em horas e minutos) eles haviam dedicado a 11 tipos diferentes de atividades de procura: conversando com amigos e parentes, lendo artigos em livros e revistas, lendo/ouvindo propaganda em televisão e rádio, lendo artigos em revistas especializadas, lendo brochuras/panfletos de fabricantes de automóveis, procurando informações sobre carros na Internet, dirigindo para/ de concessionárias de automóveis, visitando showrooms, conversando com vendedores de automóveis, negociando preço com as concessionárias e realizando testes de veículos. Também foi solicitado aos respondentes informarem quantos testes de veículos eles haviam feito. Uma vez que esta medida se mostrou mais consistente do que a estimativa de tempo utilizada em testes de veículos (presumivelmente, é mais fácil lembrar quantos testes de veículo foram feitos do que quanto tempo foi gasto nesta atividade), o tempo gasto com testes de veículos foi estimado multiplicando-se o número de testes de veículos efetivamente realizados pelo tempo médio obtido da amostra gasto em cada teste. O tempo informado em todas estas atividades foi acumulado em uma única variável $(t)$.

\section{Metodologia para a Coleta de Dados}

Em fevereiro de 2000 foram enviados pelo correio 3.000 questionários impressos em papel para uma amostra de indivíduos residentes na Região Metropolitana de Buffalo (Nova Iorque, EUA) e que haviam comprado um veículo novo durante o verão (corresponde aos meses de junho a setembro) de 1999. Esta amostra de indivíduos, escolhida por critério de acesso, foi obtida aleatoriamente a partir dos registros da Autoridade de Trânsito do Estado de Nova Iorque. O período médio de seis meses decorrido entre a compra e a pesquisa é típico em estudos envolvendo procura por automóveis (Punj e Staelin, 1983; Ratchford e Srinivasan, 1993).

Foram enviadas duas cartas, reforçando o pedido para preenchimento dos questionários, sendo uma cerca de duas semanas e outra cerca de quatro semanas após o envio dos questionários.

O questionário original, dividido em sete seções, distribuídas em onze páginas, tinha por objetivo coletar informações sobre vários aspectos do processo de decisão de compra de um automóvel novo. Assim, apenas uma parcela das informações obtidas por meio deste questionário foi utilizada neste estudo. Especificamente, foram analisadas apenas as informações referentes às variáveis descritas na seção anterior.

Foram recebidos 886 questionários, correspondendo a uma taxa de retorno de 
29,5\%, considerada típica para estudos envolvendo comportamento do consumidor; 91 questionários foram eliminados da análise por não conterem respostas para uma ou mais das variáveis a serem analisadas, reduzindo o número de questionários analisados a 795. Ao se analisar os 91 questionários eliminados, observou-se que as respostas em branco estavam distribuídas homogeneamente entre todas as variáveis incluídas neste estudo. Da mesma forma, não se observaram diferenças significativas em relação à nenhuma das variáveis utilizadas neste estudo entre os questionários eliminados da análise e aqueles que foram efetivamente utilizados.

\section{Resultados}

Dada a natureza do bem pesquisado, era de se esperar que o perfil da amostra tivesse algumas diferenças em relação ao perfil médio da população dos Estados Unidos. Especificamente, o respondente médio da amostra é mais velho, tem maior nível de escolaridade e renda familiar superior à do cidadão norte-americano médio; a amostra também continha uma proporção maior do que na população de homens e pessoas casadas (vide Tabela 1).

\section{Tabela 1: Comparação de Determinadas Características Demográficas da Amostra x População dos EUA}

\begin{tabular}{|l|l|l|}
\hline \multicolumn{1}{|c|}{ Característica Demográfica } & \multicolumn{1}{|c|}{ Amostra } & População (dados referentes a 1999) \\
\hline Até 30 anos de idade & $12,8 \%$ & $41,9 \%$ (a) \\
\hline Grau de Escolaridade & $\begin{array}{l}48,7 \% \text { completou o nível superior e } \\
25,0 \% \text { completou pelo menos 1 ano } \\
\text { de pós-graduação }\end{array}$ & $\begin{array}{l}25,5 \% \text { completou pelo menos 4 anos } \\
\text { de nível superior }\end{array}$ \\
\hline Casados & $67,3 \%$ & $54,3 \%$ \\
\hline Homens & $51,2 \%$ & $48,8 \%$ \\
\hline Renda Familiar & Aproximadamente $\$ 66.500$ & Aproximadamente $\$ 54.800$ \\
\hline
\end{tabular}

Fonte: U.S. Census Bureau (200?).

Nota: (a) dados referentes à população com até 29 anos de idade.

O preço médio pago pelo carro foi US\$21,348 e o desconto médio obtido foi US $\$ 1,639$ (7,7\% do preço médio), sendo US $\$ 1,018$ dados pelo fabricante e US\$621 dados pela concessionária; $43,5 \%$ dos respondentes utilizaram o carro usado como parte do pagamento, que foi valorizado em média em US\$5,094.

Em média, os respondentes consideraram 2,8 modelos e 2,3 concessionárias. O tempo total médio de procura foi 16,9 horas, sendo 1,5 hora na Internet e 15,4 horas em outras fontes. A Internet foi consultada por 39,6\% dos respondentes. 


\section{Tempo de Procura e Uso da Internet}

Contrariando a terceira hipótese, os compradores que consultaram a Internet investiram 20,7 horas em procura, enquanto os compradores que não consultaram a Internet investiram apenas 14,4 horas em procura ( $\mathrm{t}=-4.577, \mathrm{p}<0,01)$. O usuário da Internet utilizou, em média, 3,8 horas consultando a Internet e 16,9 horas procurando em outras fontes de informações. Portanto a razão da diferença entre o tempo investido em procura pelos dois grupos na procura não se deveu exclusivamente ao tempo adicional utilizado na Internet, já que o usuário da Internet também procurou por mais tempo em outras fontes (16,9 versus $14,4 ; \mathrm{t}=-2,030, \mathrm{p}<0,05)$.

Outro forte indicador do tempo investido em procura por automóveis é o número de testes de veículos que os potenciais compradores fazem: verificou-se que o usuário da Internet realizou, em média, 2,9 testes de veículos, enquanto os compradores que não consultaram a Internet realizaram apenas 2,2 testes $(\mathrm{t}=-4,762, \mathrm{p}<0,01)$.

Este resultado não chegou a ser surpreendente, já que também havia sido sugerido que os usuários da Internet possuem um perfil diferente dos não usuários. De fato, a diferença entre os dois grupos foi semelhante à diferença verificada na população norte-americana. Especificamente, os compradores que consultaram a Internet na amostra são mais jovens (41 anos versus 52 anos; $\mathrm{t}=11,657, \mathrm{p}<0,01$ ), têm maior escolaridade (15,9 anos de escolaridade versus 14,$6 ; \mathrm{t}=-6,872, \mathrm{p}<0,01)$ e têm renda familiar superior ( $\$ 76,107$ versus $\$ 59,898$; $\mathrm{t}=-5,053$, $\mathrm{p}<0,01$ ).

Também como era esperado, verificou-se que os usuários da Internet possuem um conjunto de preferências maior e mais complexo. Especificamente, os usuários que consultaram a Internet compraram veículos mais caros $(\$ 21,861$ versus $\$ 20,984 ; \mathrm{t}=-1,652, \mathrm{p}<0,1)$, compraram veículos com mais opcionais $(9,6$ versus $9,3 ; \mathrm{t}=-2,177, \mathrm{p}<0,05)$, consideraram mais modelos de veículos (3,0 versus 2,6; $\mathrm{t}=-4,489, \mathrm{p}<0,01)$ e mais concessionárias $(2,5$ versus 2,$2 ; \mathrm{t}=-4,585, \mathrm{p}<0,01)$ e procuraram o veículo por mais tempo (4,2 meses versus 3,5 meses; $t=-3,059$; $\mathrm{p}<0,01)$.

Assim, o fato de os compradores que consultaram a Internet investirem mais tempo com procura do que aqueles que não consultaram a Internet, pode ser mais bem explicado pela diferença entre os perfis dos dois grupos. Portanto, embora H3 deva ser rejeitada, as razões que levaram os usuários da Internet a procurar por mais tempo se justifica por diferenças individuais, conforme havia sido sugerido. 


\section{Descontos Obtidos na Compra de Veículos Novos e Uso da Internet}

A primeira hipótese afirma que o tempo investido em procura está positivamente associado à obtenção de maiores descontos e a segunda hipótese afirma que os compradores de veículos novos que consultaram a Internet obtiveram descontos maiores do que os compradores que não consultaram a Internet. Para testar estas duas hipóteses, resolveu-se o sistema de equações proposto, utilizando procedimento similar àquele utilizado no Método de Dois Estágios empregado com equações simultâneas, contendo variáveis dependentes comuns. As variáveis HOMEM e CASADO foram utilizadas apenas como instrumentos durante o primeiro estágio; as variáveis $D$ e $t$ foram consideradas como variáveis endógenas e as demais variáveis ( $V$ e $C$ ) foram consideradas exógenas.

Da mesma forma como foi feito em Ratchford e Srinivasan (1993), seguindo procedimento sugerido por Anderson e Weitz (1989), as equações (1) e (2) foram estimadas sem as variáveis dummy correspondentes aos modelos de veículos. A seguir, foram incluídas na estimativa destas duas equações apenas as variáveis dummy de modelos que estavam significativamente correlacionadas com os erros obtidos no passo anterior. Das 124 variáveis correspondentes a modelos de veículos, 15 foram incluídas como variáveis exógenas na equação (1) e 19 na equação (2). Isto significou incorporar 32 variáveis exógenas na estimativa das variáveis instrumento $t$ e $D$. Todas as variáveis de modelos incluídas por meio deste procedimento foram consideradas significativas ao nível de 10\% de confiança nas estimativas finais das equações (1) e (2).

Uma vez que o logaritmo de zero não está definido, também foi necessário adicionar uma hora aos valores declarados de procura total, procedimento muito comum em análises de regressão de logaritmos (por exemplo, em Choe e Jeong [1993]).

O resumo dos resultados obtidos com as estimativas do coeficientes das equações (1) e (2), tanto no primeiro estágio quanto no segundo estágio, estão descritos na Tabela 2.

O coeficiente de explicação ajustado $\left(\mathrm{R}^{2}\right)$ de 0,37 obtido para as estimativas de $t$ está um pouco abaixo de estudos similares (Ratchford e Srinivasan [1993] obtiveram um coeficiente de 0,43; Urbany, Dickson e Kalapurakal [1996] obtiveram um coeficiente de 0,45 ). $O$ teste de análise de variância, entretanto, revela que o modelo é significativo, ou seja, a hipótese de que todos os coeficientes são iguais a zero é rejeitada $(\mathrm{F}=13,618, \mathrm{p}<0,01)$. 


\section{Tabela 2: Resumo dos Resultados das Regressões}

\begin{tabular}{|c|c|c|c|c|c|c|c|c|}
\hline \multirow[t]{2}{*}{$\begin{array}{c}\text { Variáveis } \\
\text { Independentes }\end{array}$} & \multicolumn{2}{|c|}{$\begin{array}{l}1^{\circ} \text { Estágio } \\
\log t \text { (a) }\end{array}$} & \multicolumn{2}{|c|}{$\begin{array}{l}1^{\circ} \text { Estágio } \\
\log D \text { (a) }\end{array}$} & \multicolumn{2}{|c|}{$\begin{array}{l}2^{\circ} \text { Estágio } \\
\log t \text { (b) }\end{array}$} & \multicolumn{2}{|c|}{$\begin{array}{l}2^{\circ} \text { Estágio } \\
\log D \text { (c) }\end{array}$} \\
\hline & C.E. & D.P. & C.E. & D.P. & C.E. & D.P. & C.E. & D.P. \\
\hline Intercepto & $1,889^{* * *}$ & ,205 & $-68,124$ & 41,305 & $1,861^{* * *}$ & , 197 & $-14,767$ & 10,080 \\
\hline $\log D$ & & & & &,- 0002 & ,011 & & \\
\hline $\log t$ & & & & & & & ,2834 & 1,037 \\
\hline CINTER & $196^{* * *}$ & ,054 &,$- 9353^{* *}$ & ,421 &, $184^{* * *}$ & ,055 &,$- 9793^{* *}$ & ,461 \\
\hline MODVEIC &, $115^{* * *}$ & ,024 &, $4939^{* * * *}$ & , 183 &, $114^{* * * *}$ & ,025 & $4735^{* *}$ & ,221 \\
\hline CONCESS & $.226^{* * *}$ & , 027 & , 1437 & ,204 &, $224^{* * *}$ & ,026 & , 1113 & ,3121 \\
\hline NCARDEZ &,$- 0042^{* *}$ &, 018 & ,0853 & , 136 &,$- 005^{* * *}$ &, 017 & , 1787 & , 144 \\
\hline NCONCDEZ &, $0077^{* * *}$ &, 025 &,$- 3643^{*}$ & , 191 &, $0074^{* * * *}$ & ,024 &,$- 353^{*}$ & ,208 \\
\hline QUANDO1 &,$- 587^{* * *}$ &, 115 & 1,027 & ,888 &,$- 552^{* * *}$ &, 114 & ,755 & 1,050 \\
\hline QUANDO2 &,$- 300^{* * *}$ &, 110 & 1,1659 & ,850 &,$- 287^{* * * *}$ & , 109 & 8895 & 897 \\
\hline QUANDO3 &,$- 253^{* *}$ &, 114 & ,8978 & ,876 &,$- 224^{* *}$ &, 113 & ,7658 & ,905 \\
\hline QUANDO4 &,- 179 & , 119 & 1,1907 & ,915 &,- 165 & , 118 & ,9976 & ,931 \\
\hline CERTOMNAO &, $264^{* *}$ &, 120 & ,0989 & ,924 &, $243^{* *}$ & , 118 &,- 1312 & ,954 \\
\hline CERTOMSIM &,- 00729 & ,058 &,- 6819 & ,443 &,- 0094 & ,058 &,$- 9341^{*}$ & ,461 \\
\hline CERTOFNAO &,- 00084 & , 070 & ,3427 & ,538 &,- 00248 & ,069 & ,3079 & ,548 \\
\hline CERTOFSIM &,- 00574 & ,066 & $1,386^{* * *}$ & ,517 &,- 00464 & ,068 & $1,393^{* *}$ &, 529 \\
\hline OPTOTAL & ,00157 & ,016 &, 1173 & ,121 &,- 00107 & ,015 & , 1608 & ,123 \\
\hline USADO &,- 00218 &, 048 & $-2,66^{* * *}$ &, 376 &,- 00139 &, 054 & $-2,55^{* * *}$ & ,380 \\
\hline POSGRAD &,- 00087 & ,069 &,- 5447 & ,537 &,- 00112 & ,068 &,- 537 & ,544 \\
\hline GRADUADO &,- 00340 & ,060 &,- 2350 & ,469 &,- 0033 & ,059 &,- 354 & ,479 \\
\hline IDADE30 &, $232^{* *}$ & ,094 &,- 6170 & ,729 & $266^{* * *}$ & ,090 & ,0119 & ,766 \\
\hline IDADE3140 & ,00534 & ,079 & ,266 & ,621 & ,006921 & ,077 & ,3549 & ,619 \\
\hline IDADE4150 & ,112 & , 071 & $-1,368^{* *}$ & ,552 & , 110 & ,070 & $-1,161^{* *}$ & .559 \\
\hline IDADE5160 &, 0016 &, 076 &,- 249 & ,594 &, 00325 &, 074 &,- 4772 & ,589 \\
\hline HOMEM &,- 00589 &, 050 &,- 4696 & ,385 & & & & \\
\hline CASADO &,$- 0099^{*}$ &, 055 & $-1,13^{* * *}$ &, 428 & & & & \\
\hline $\begin{array}{l}\mathrm{R}^{2} \\
\text { McFadden's } \mathrm{R}^{2} \\
\mathrm{~N}\end{array}$ & & ,370 & & $\begin{array}{r}, 057 \\
795\end{array}$ & & , 370 & & $\begin{array}{r}, 048 \\
795\end{array}$ \\
\hline
\end{tabular}

Nota: C.E = coeficiente estimado. D.P. desvio-padrão da estimativa (a) contém 32 variáveis dummy correspondentes aos modelos de veículos, todas significativas ao nível de confiança de 10\%; (b) contém 15 variáveis dummy para modelos, todas significativas ao nível de confiança de 10\%; (c) contém 19 variáveis dummy para modelos, todas significativas ao nível de confiança de 10\%.

*** Significativo ao nível de confiança de $1 \%$. ${ }^{* *}$ Significativo ao nível de confiança de 5\%. ${ }^{*}$ Significativo ao nível de confiança de $10 \%$.

Para se comparar os resultados da equação (2) com outros estudos, calculouse o coeficiente de explicação sugerido por McFadden (1974), obtendo-se um índice de 0,048 , que pode ser comparado aos índices próximos a 0,18 obtidos por Goldberg (1996). Ressalta-se, entretanto, que o número de variáveis explicativas incluídas naquele estudo é muito superior ao número de variáveis incluídas neste estudo. Além disso, como o interesse principal era o efeito da utilização da Internet como fonte de informações sobre os descontos obtidos, apenas a inclusão desta 
variável seria suficiente para os propósitos deste estudo. O Teste de Proporção de Probabilidade revelou que a hipótese de que os coeficientes da equação são iguais a zero é rejeitada $(D=-2 \mathrm{LLR}=178 ; \mathrm{p}<0,01)$.

Os resultados obtidos para a regressão da equação (1) indicam que o tempo de procura: (a) é maior para as pessoas que consultaram a Internet, conforme observado na seção anterior; (b) aumenta com o aumento do conjunto evocado de marcas e fabricantes, o que está de acordo com as previsões; (c) diminui com o aumento de carros adquiridos nos últimos dez anos, de acordo com as previsões; (d) aumenta com o número de concessionárias contatadas nos últimos dez anos, o que contraria as previsões; (e) aumenta com o tempo que o comprador levou no processo de compra, conforme as previsões; (f) é maior para pessoas que não estavam certas em relação à marca do veículo, conforme previsto; (g) é maior para pessoas com até 30 anos de idade; os resultados, entretanto, não foram significativos para os outros grupos de idade, apoiando parcialmente as previsões.

Os resultados também não foram significativos para as demais variáveis. O que talvez mais chame a atenção é o fato de que os descontos não influenciaram, de nenhuma forma, o tempo de procura. Este resultado sinaliza que a variável $D$ não está correlacionada com $\varepsilon_{2}$ em (2), contrariando a hipótese de interdependência entre $D$ e $t$ e levando à rejeição de H1.

Para que H2 fosse aceita, o coeficiente da variável CINTER na equação (2) teria de ser positivo. Entretanto, ao contrário do que havia sido previsto, os resultados indicam que os compradores que consultaram a Internet obtiveram menores descontos.

A análise dos resultados obtidos para a regressão da equação (2) indica que os descontos obtidos: (a) são menores para as pessoas que consultaram a Internet, contrariando a hipótese; (b) aumenta com o aumento do conjunto evocado de marcas; (c) diminui com o aumento do número de concessionárias contatadas nos últimos dez anos, contrariando as previsões; (d) é menor para as pessoas que estavam certas em relação à marca desejada, confirmando as previsões; (e) é maior para as pessoas que estavam certas em relação à concessionária, também confirmando a hipótese; (f) é menor para as pessoas que deram seus carros usados como parte do pagamento, o que também confirma as previsões; (g) é menor para as pessoas com idade entre 41 e 50 anos, embora os resultados não sejam significativos para outros grupos de idades.

Além disso, o fato de o coeficiente para a variável $t$ não ser significativo na equação (2), confirma que H1 deva ser rejeitada. 


\section{Discussão}

Como vimos na seção anterior, os resultados indicam que o fato de um comprador ter consultado a Internet não contribuiu para a obtenção de maiores descontos. Além disso, também se verificou que o maior tempo de procura não contribuiu significativamente para a obtenção de maiores descontos. No entanto, como havia sido constatado, o perfil do usuário da Internet é significativamente diferente do perfil do não usuário da Internet. O usuário da Internet, além de ser mais jovem, o que pode implicar menos experiência e maior procura, também tem renda superior ao não usuário, o que pode sugerir que ele é menos sensível a preços, justificando o menor nível de descontos. Caso estas hipóteses estejam corretas, ao se analisar os dois grupos em conjunto, pode-se ter deixado de observar efeitos que seriam observados se os dois grupos fossem analisados separadamente.

Ao se estimar os coeficientes da segunda equação para os dois grupos separadamente, observaram-se resultados substancialmente diferentes para o grupo de usuários da Internet daqueles obtidos anteriormente, considerando os dois grupos juntos.

Especificamente, para o grupo que consultou a Internet, percebe-se que os descontos: (a) aumentam com o tempo de procura; (b) aumentam com a certeza da concessionária; (c) diminuem com a certeza do fabricante; (d) diminuem para aqueles que deram o carro usado como parte do pagamento. Portanto a hipótese de que o tempo de procura está associado à obtenção de maiores descontos não pode ser rejeitada para o grupo de usuários da Internet.

No entanto, para o grupo que não consultou a Internet, os descontos: (a) aumentam com o aumento do conjunto evocado de marcas; (b) diminuem com o número de concessionárias contatadas nos últimos dez anos; (c) aumentam para as pessoas que levaram entre 1 e 3 meses no processo de compra; (d) aumentam para as pessoas que têm entre 41 e 50 anos de idade; (e) diminuem para as pessoas que deram seu carro obtido como parte do pagamento. O coeficiente estimado de $t$ não foi significativo e, portanto, deve-se rejeitar a hipótese de que o tempo de procura está positivamente associado à obtenção de maiores descontos.

Ainda assim, observa-se que estes resultados devem ser vistos com muita cautela. Apesar de se rejeitar a hipótese de que os coeficientes sejam diferentes de zero para as equações de descontos dos dois grupos - usuários e não usuários da Internet $-(\mathrm{D}=-2 \mathrm{LLR}=59,34,27$ g.l., $\mathrm{p}<0,01$ e $\mathrm{D}=-2 \mathrm{LLR}=127,68,32$ g.l., $\mathrm{p}<0,01$, respectivamente), os coeficientes de explicação, utilizando o método 
proposto por McFadden (1974), para as duas equações são relativamente baixos (0,0395 e 0,058, respectivamente).

Uma vez que os dados deram pouco suporte à hipótese de que o tempo investido em procura está associado positivamente à obtenção de maiores descontos, voltase para a seguinte questão: Será que o tempo de procura está associado positivamente com maior satisfação na compra?

O questionário solicitou aos respondentes dar uma nota, variando de 1 a 7 , a 13 características do carro adquirido (por exemplo, capacidade de carga, consumo de combustível, preço do carro etc.). Também foi solicitado aos respondentes distribuir 100 pontos às mesmas 13 características, de acordo com a sua importância. O mesmo foi feito em relação a 8 características da concessionária onde o veículo foi adquirido (por exemplo, localização conveniente, atratividade dos veículos que a concessionária vende, disponibilidade de veículos no pátio etc.).

A partir destas medidas, foram construídas duas medidas de satisfação: a primeira, multiplicando-se a nota dada à cada característica do veículo adquirido pela importância relativa da característica; a segunda, multiplicando-se a nota dada a cada característica da concessionária pela importância relativa da característica. É razoável supor que estas duas medidas poderiam representar uma medida de satisfação com a compra.

Para testar a hipótese de que o maior tempo de procura estava associado positivamente à maior satisfação com o carro adquirido e com a concessionária, calculou-se o índice de correlação de Pearson entre as duas variáveis de satisfação e o tempo total de procura. Apesar de ter sido observada uma correlação positiva entre a satisfação com o carro adquirido e a satisfação com a concessionária onde o carro foi adquirido ( $p=0,234$; $p<0.01$ ), não se observou nenhuma correlação significativa entre estas duas medidas de satisfação e o tempo de procura.

Desta forma, pode-se afirmar que a hipótese de que o maior tempo de procura está associado à maior satisfação com a compra, pode ser negada.

\section{Conclusão}

Este estudo foi motivado pelo crescente debate sobre a utilização da Internet como agente intermediário entre compradores e consumidores. Portanto, além de tentar replicar estudos anteriores sobre os benefícios obtidos com a procura, 
este estudo também se propôs a verificar se os consumidores que utilizaram a Internet obtiveram benefícios maiores do que aqueles que não a utilizaram.

Os resultados para uma amostra representativa de uma região dos Estados Unidos indicam que os consumidores que consultaram a Internet procuraram por mais tempo do que aqueles que não consultaram a Internet. No entanto os resultados de análises adicionais mostraram que o perfil dos usuários da Internet difere significativamente do perfil dos não usuários da Internet.

O modelo também permitiu identificar diversas variáveis determinantes do tempo de procura por automóveis, sendo que estas variáveis, tomadas em conjunto, foram responsáveis por aproximadamente um terço da variância observada na variável tempo de procura. A maioria das hipóteses sobre o efeito destas variáveis foi aceita (das 13 hipóteses, 6 foram aceitas totalmente, 2 parcialmente e 5 foram rejeitadas).

Ao se analisarem os dois grupos em conjunto (usuários e não usuários da Internet), verificou-se que os compradores que consultaram a Internet obtiveram menores descontos do que aqueles que não a consultaram e que o tempo de procura não teve nenhuma influência sobre os descontos obtidos na compra de automóveis novos. No entanto, na análise dos dois grupos separadamente, constatou-se que o tempo de procura influiu positivamente na obtenção de descontos maiores para o grupo de usuários da Internet, mas não para o grupo de não usuários. Vale ressaltar, entretanto, que estes resultados devem ser vistos com cautela, principalmente levando-se em conta que as variáveis incluídas no modelo foram responsáveis por uma pequena parcela da variância observada na variável dependente $D$.

Também não foi possível observar na amostra uma associação positiva entre o tempo investido na procura e a satisfação com o veículo adquirido e com a concessionária na qual o veículo foi adquirido, embora tenha sido observada uma associação positiva entre as duas medidas de satisfação.

Deve-se ressaltar que este estudo possui algumas limitações. A primeira limitação refere-se ao fato de não ter sido possível o acesso a dados de oferta, como por exemplo, volume de estoques das concessionárias e grau de concorrência. Este fato pode ter sido parcialmente responsável pelos baixos coeficientes de explicação obtidos para a equação de descontos. Em mercados não perfeitamente competitivos, os preços tendem a ser fortemente influenciados pela oferta, neste caso pelas concessionárias. Estudos futuros devem considerar a inclusão destes tipos de dados na análise. 
Outra limitação refere-se ao fato de não ter sido possível a utilização de dados referentes à renda pessoal ou familiar. $\mathrm{O}$ número de respostas em branco para estas duas variáveis foi muito alto (33,6\% e 7,7\%, respectivamente) e nenhum dos métodos empregados para estimar as respostas em branco obteve sucesso. A renda pessoal, além de proporcionar uma medida do custo de oportunidade do tempo, também é um bom indicador dos preços de reserva de cada indivíduo.

Vale lembrar que as compras deste estudo foram feitas no mercado convencional e que a Internet foi utilizada apenas como fonte de informações. Futuros estudos devem considerar a possibilidade de comparar as compras de automóveis realizadas por meio da Internet com aquelas realizadas em concessionárias convencionais.

É possível também que o modelo utilizado aqui não tenha sido capaz de captar o efeito das variáveis independentes sobre os descontos obtidos. Estudos futuros devem considerar formulações alternativas. Uma vez que é razoável supor que as pessoas, que não conseguiram descontos, foi porque eventualmente não os pediram, uma alternativa seria considerar a obtenção de descontos como um processo de dois estágios: no primeiro estágio, analisa-se exclusivamente a decisão de o comprador pedir ou não o desconto; no segundo estágio, analisa-se o montante de descontos obtidos apenas daqueles que efetivamente obtiveram algum desconto. Este tipo de formulação tem sido utilizado em marketing para analisar o volume de compras de determinadas marcas com base em dados seriados (Doyle, 1977; Guadagni e Little, 1983). Esta formulação implicaria considerar a variável $D$ como variável truncada e não censurada, como feito aqui. Além disto, seria necessário também estimar a decisão sobre pedir ou não descontos por meio de um Modelo Probit.

Finalmente, é bom lembrar que os dados têm natureza seccional e são representativos apenas de uma região dos Estados Unidos. Apesar disto, acreditase que os resultados deste estudo possam ser extrapolados para um universo maior, se for levado em consideração o tamanho da amostra e as características da região de Buffalo: alta densidade populacional, caracteristicamente urbana e altamente desenvolvida. Além disso, considerando-se o arcabouço teórico utilizado neste estudo, não se acredita que o contexto no qual a pesquisa foi realizada possa ter tido qualquer influência sobre os resultados obtidos; resultados semelhantes poderiam ser esperados, caso o contexto fosse outro. Dada a importância que a Internet vem adquirindo no Brasil, estudos futuros devem considerar a utilização de dados coletados no Brasil para diferentes categorias de produto. 


\section{Notas}

${ }^{1}$ Agradeço ao Prof. Brian Ratchford (University of Maryland) os conselhos para a elaboração deste artigo, ao Prof. Robert Krapfel (University of Maryland) e ao Prof. Rubens da Costa Santos (EAESP-FGV) o apoio durante o período em que estive visitando a Universidade de Maryland e aos revisores anônimos desta revista. Este artigo contou com o apoio da CAPES.

${ }^{2}$ Em 2000, apenas 326.000 norte-americanos compraram seus carros pela Internet, comparado com os mais de 17 milhões que preferiram as vias convencionais (Time, 2001).

${ }^{3} \mathrm{O}$ primeiro sinal indica o tipo de influência da variável independente sobre o tempo de procura; o segundo sinal indica o tipo de influência sobre os descontos obtidos.

${ }^{4}$ Uma modalidade de desconto muito comum no mercado de automóveis norte-americano é o chamado rebate. Nesta modalidade de desconto, o comprador paga o preço total à concessionária e depois é reembolsado diretamente pelo fabricante. Desta forma, ao contrário do mercado de automóveis brasileiro, o comprador sabe exatamente qual o desconto conseguido tanto do fabricante quanto da concessionária.

\section{ReferênCias Bibliográficas}

ANDERSON, E.;

WEITZ, B.

Determinants of continuity in conventional industrial channels dyads. Marketing Science, v. 8, p. 310-323, Fall 1989.

\section{AYRES, I.}

Fair driving: gender and race discrimination in retail car negotiations. Harvard Law Review, v. 10, p. 817-872, Feb. 1991.

AYRES, I.;

\section{SIEGELMAN, P.}

Race and gender discrimination in bargaining for a new car. The
American Economic Review, v. 85, n. 3, p. 305-322, 1995.

BAKOS, J. Y.

Reducing buyer search costs: implications for electronic marketplaces. Management Science, v. 43, n. 12, p. 1676-1692, 1997.

BEATTY, S. E.;

SMITH, S. M.

External search effort: an investigation across several product categories. Journal of Consumer Research, v. 14, n. 1, p. 83-95, 1987. 
BLAYLOCK, J. R.;

SMALLWOOD, D. M.

Intrahousehold time allocation: the case of grocery shopping. Journal of Consumer Affairs, v. 21, p. 183-201, Winter 1987.

BRUCKS, $\mathrm{M}$.

The effects of product class knowledge on information search behavior. Journal of Consumer Research, v. 12, p. 1-16, June 1985.

BRYNJOLFSSON, E.;

SMITH, M. D.

Frictionless commerce? A comparison of internet and conventional retailers.

Management Science, v. 46, n.

4, p. 563-585, 2000.

CARLSON, J. A.;

GIESEKE, R. J.

Price search in a product market.

Journal of Consumer

Research, v. 9, n. 4, p. 357-365, 1983.

CHOE, Y. S.;

JEONG, J.

National Tax Journal, v. 46, n.

1, p. 33-39, 1993.

DEGERATU, A. M.;

RANGASWAMY, A.;

WU, J.

Consumer choice behavior in online and traditional supermarkets: the effects of brand name, price, and other search attributes. International Journal of Research in Marketing, v. 17, p. 55-78, Mar. 2000.

DOYLE, P.

The application of probit, logit, and tobit in marketing: a review. Journal of Business Research, v. 5, p. 235-248, Sept. 1977.

FEICK, L.;

PRICE, L.

The market maven: a diffuser of marketplace information. Journal of Marketing, v. 51, p. 83-97, Jan. 1987.

FINDING a car online.

Time, 05 feb. 2001. Edição Especial, p. 42-50.

GOLDBERG, P. K.

Dealer price discrimination in new car purchases: evidence from the consumer expenditure survey. Journal of Political Economy, v. 104, n. 3, p. 622-654, 1996.

GUADAGNI, P. M.;

LITTLE, J. D. C.

A logit model of brand choice calibrated on scanner data. Marketing Science, v. 2, n. 3, p. 203-238, 1983. 
KLEIN, L. R.;

FORD, G. T.

Consumer search for information in the digital age: an empirical study of pre-purchase search for automobiles. In: ASSOCIATION FOR CONSUMER RESEARCH, Austin, 2001. Proceedings... Austin: [s.n.], 2001.

\section{LEE, H. G.}

Do electronic marketplaces lower the price of goods? Communications of the ACM, v. 41, n. 1, p. 73-80, 1998.

LYNCH JR., J. G.;

ARIELY, D.

Wine online: search costs affect competition on price, quality, and distribution. Marketing Science, v. 19, n. 1, p. 83-103, 2000.

MARMOSTEIN, H.;

GREWAL, D.;

FISHE, R. P.

The value of time spent in pricecomparison shopping: survey and experimental evidence. Journal of Consumer Research, v. 19, p. 154-165, June 1992.

\section{MCFADDEN, D.}

Conditional logit analysis of qualitative choice behavior. In: ZAREMBKA, P. (Ed.). Frontiers in econometrics. New York: Academic Press, 1974. p. 105-142.
MORTON, F. S.;

ZETTELMEYER, F;

RISSO, J. S.

Internet car retailing. Yale University, UC Berkeley and J.D. Power Associates, Dec. 2000. working paper.

NELSON, F.;

OLSON, L.

Specification and estimation of a simultaneous-equation model with limited dependent variables. International Economic Review, v. 19, n. 3, p. 695-709, 1978.

NEWMAN, J.;

STAELIN, R.

Repurchase information seeking for new cars and major household appliances. Journal of Marketing Research, v. 9, n. 3, p. 249, 1972.

PUNJ, G. N.;

STAELIN, R.

A model of consumer information search behavior for new automobiles. Journal of Consumer Research, v. 9, n. 4, p. 366-380, 1983.

RATCHFORD, B.

Cost-benefit models for explaining consumer choice and information seeking behavior. Management Science, v. 28, p. 197-212, Feb. 1982. 
RATCHFORD, B.; SRINIVASAN, N.

An empirical investigation of returns to search. Marketing Science, v. 12, n. 1, p. 73-87, 1993.

RATCHFORD, B. T.;

TALUKDAR, D.;

LEE, M. S.

A model of consumer choice of the internet as an information source. [S.l.], 2000. working paper.

SCHMIDT, J. B.;

SPRENG, R. A.

A proposed model of external consumer information search.

Journal of the Academy of Marketing Science, v. 23, p. 246-256, Summer 1996.

SMITH, M. D.;

BAILEY, J.;

BRYNJOLFSSON, E.

Understanding digital markets: review and assessment. In: BRYNJOLFSSON, E.; KAHIN, $B$. (Eds). Understanding the digital economy. New York: MIT Press, 1999.
SRINIVASAN, N.;

RATCHFORD, B.

An empirical test of a model of external search for automobiles.

Journal of Consumer Research, v. 18, p. 233-242, Sept. 1991.

STIGLER, G. J.

The economics of information. Journal of Political Economy, v. 69, p. 213-225, June 1961.

TOBIN, J.

Estimation of relationships for limited dependent variables. Econometrica, v. 26, p. 24-36, 1958.

URBANY, J. E.; DICKSON, P. R.; KALAPURAKAL, R.

Price search in the retail grocery market. Journal of Marketing, v. 60, p. 91-104, Apr. 1996.

VARIAN, H. R.

Microeconomic analysis. 2. ed. New York: Norton, 1984. 